\title{
Measuring behavior in sport and exercise
}

\author{
Tom Allen ${ }^{1}\left[\right.$. Robyn Grant $^{2} \cdot$ Stefan Mohr ${ }^{3} \cdot$ Jonathan Shepherd ${ }^{4,5}$
}

Accepted: 9 November 2020 / Published online: 25 November 2020

(c) International Sports Engineering Association 2020

Whether it is how equipment interacts with the human body, or how a player perceives equipment, sports equipment development is ultimately linked to the measurement of human behavior. Indeed, measuring behavior is key to sports equipment design and uptake. Measuring behavior is different from the mature discipline of sports biomechanics, since it focuses specifically on the methodological development of measurement techniques, with a close alignment to engineering and technology. As well as being methods based, measuring behavior has a broad scope, including physiological, psychological or movement measurement systems, all of which are pertinent to the field of sports engineering. Subsequently, when we hosted the 11th International Conference of Measuring Behavior, in June 2018 at Manchester Metropolitan University, sport was included as one of the main themes for the conference for the first time. This theme was the focus of the final day of the conference, which started with a keynote lecture on the classification of swimmers with physical impairments, followed by two sessions on sport. These sessions were on: (1) Measuring behavior in elite sport and (2) Monitoring and encouraging participation in sport and physical activity (see Conference Proceedings [1]). Following these sessions, the conference concluded with a visit to the National Cycling Centre in Manchester, hosted by British Cycling. These activities were funded by an Engaging Sports Engineering Grant from the ISEA (International Sports Engineering Association).

Tom Allen

t.allen@mmu.ac.uk

1 Department of Engineering, Manchester Metropolitan University, Manchester, United Kingdom

2 Department of Natural Sciences, Manchester Metropolitan University, Manchester, United Kingdom

3 HEAD Sport GmbH, Wuhrkopfweg 1, 6921 Kennelbach, Austria

4 Ping, Inc, 2201 W. Desert Cove, Phoenix, Arizona, United States

5 Griffith University, Brisbane, Australia
Following on from the success of Measuring Behavior 2018 and in response to the high level of research in this area, we released a call for papers for this Topical Collection on Measuring Behavior in Sport and Exercise. The intention of the call was to capture work presented at Measuring Behavior 2018 as well as other studies on the broad theme of measuring behavior for sport and exercise. The collection includes nine articles on topics ranging from flow visualisation around swimmers to virtual reality training for karate athletes. Three of the articles include work presented at Measuring Behavior 2018, and these focus on measuring: (1) racewalking movement patterns, (2) wrist joint angles in boxing and (3) emotions during ziplining. This editorial summarises the topical collection and presents our thoughts on the future of behavioral measurement in sport and exercise.

Advanced measurement techniques can help us to further our understanding of sports mechanics. van Houwelingen et al. developed techniques to visualise the flow behind an underwater swimmer. Air bubbles released through the base of a swimming pool were filmed with six synchronised cameras to form an underwater measurement volume. Footage of a swimmer passing through the volume was analysed using particle image velocimetry software to track the movement of the bubbles. The setup was demonstrated for a swimmer performing dolphin kicks, with vector plots and colour maps showing the trailing vortices from each down-kick. Such visual techniques offer many benefits, such as for furthering our understanding of swimming mechanics, validating computer simulations and practical experiments, athlete training, and for enhancing teaching and communication of engineering and biomechanics.

Measurement technology can also play a role in objective judging of sport and training of athletes, and the next four articles we describe cover these topics. Caporaso et al. present a tool for assessing biomechanical indices of race walkers, with potential to improve both judging and training. Data from an inertial measurement unit on the walker's lower back was used to assess biomechanical indices, including those related to ground contact, step length, and 
cadence. The indices can be presented on radar charts, so athletes, coaches, and judges can infer performance and detect infringements.

Ferger et al. present a force plate system for measuring the time of flight and the landing position of a trampolinist, with implications for judging and training. An optoelectronic sensor was placed under each leg of the trampoline to measure normal forces. An algorithm, developed previously using over 8000 jumps, extracted events from the temporal force data summed across the four plates. Local maximums corresponding to a force over $5 \mathrm{kN}$ were identified, followed by the time point each side of these where the force first fell below half the value of the local peak. Landing time was assigned to $74 \mathrm{~ms}$ before the first of these time points, with takeoff $88 \mathrm{~ms}$ after the second one. Landing position was estimated from the forces measured by the individual plates, and their known positions relative to the centre point of the trampoline. For this calculation of landing position, force readings from each plate were mean averaged across the data points exceeding half the value of the local peak. The force plate system performed well in comparison to a reference system, so it has potential as a judging aid in competitive trampolining.

Wolf et al. present techniques for providing a road cyclist with a pacing strategy that updates during a race to account for unpredictable conditions, like changes in wind speed. Before the race, a physical and physiological model provides an optimal pacing strategy, based on fixed inputs related to the course, bike, and rider. Powermeter readings can be used to calculate the remaining anaerobic resources of the cyclist during the race, with a target power displayed on a mobile device so they can make adjustments with a view to completing the course at optimal efficiency. Petri et al. present and test a virtual reality environment for training athletes in karate. To develop the system, the movements of karate experts performing attacks were recorded with an optical motion capture system. The attacks were then mapped onto avatars dressed in karate attire and located in a virtual fighting arena. The training tool was tested with 15 youth karate athletes, receiving both conventional training and virtual training with an attacking avatar. The participants wore a head-mounted display and hand targets, which were tracked with the motion capture system so their hands could be visualised in the virtual environment. Virtual reality training helped the athletes to respond faster and more effectively to karate attacks in the virtual environment.

As well as for judging and training, accurate and reliable measurement devices and techniques can be used to assess injury risk during sport. Gatt et al. investigated the suitability of an electromagnetic tracking system for measuring wrist angles in boxing. When boxers held their wrist at set angles the system was within $6^{\circ}$ of video analysis, with statistical analysis suggesting good test-retest reliability over three testing sessions. During punch testing, where the glove prevents comparison against video to assess accuracy, test-retest reliability was also good for wrist flexion-extension and substantial for radial-ulnar deviation angles. As such, electromagnetic tracking systems should be suitable for measuring joint angles during sporting movements, which could be useful for assessing interventions for reducing injury risk.

Turner et al. investigated the effect of repeated testing sessions on participant behavior. They measured the effect of golf club moment of inertia (about the handle) on both the swing and ball speed of eleven golfers over three repeated sessions. Three clubs of varying moments of inertia were tested, with about $50 \mathrm{~g}$ added at different locations on the shaft, not the head. Importantly, adding mass to the shaft ensured the overall and effective striking mass remained constant between clubs. As expected, swing and ball speed decreased as the moment of inertia increased, but the size of these effects could not be quantified due to variability between the sessions. The work has implications for researchers investigating how changes to sports equipment may influence athlete performance, where the reliability of any observations should be assessed across repeated testing sessions.

Not only can repeated sessions cause variability, but so can different participants. Jones et al. present a method for combining perpendicular strain measurements into a complex function so they can be considered jointly, allowing simple comparison of golf club shaft bending between swings. Strain gauges on the club shaft took perpendicular measurements in the lead/lag and toe/heel directions at the expected location of maximum bending. Twelve skilled golfers hit six shots with each of three clubs that varied only in shaft stiffness. Within-golfer shaft strain patterns were more consistent than those between golfers. As expected, flexible shafts bent more during the golf swing, although shaft stiffness had a smaller influence than the differences between the golfers. The work has implications for optimising the stiffness of golf club shafts, and other sporting implements, for individuals.

Wearable sensors are often used to monitor physiological responses of athletes, but it can be unclear if measured responses are driven by physical exertion, changes in emotions or both. Dupré et al. used wearable sensors to measure the physiological responses of participants, before, during, and after ziplining, an emotionally stimulating activity that requires little physical exertion. By applying Generalized Additive Mixed Models to the sensor outputs, they showed differences in physiological measures during the ziplining phases, for example, changes in heart and breathing rate and skin conductance level. The techniques presented by Dupré et al. could be used to predict the emotions of athletes during periods of low physical exertion, such as before and after 
the main activity or during breaks, with implications for performance and safety, particularly in so-called 'adventure sports' like snowboarding, mountain biking, and climbing.

We thank all authors for their contributions to this topical collection on Measuring Behavior in Sport and Exercise. The measurement methods presented here include using visual and electromagnetic tracking techniques, force data, strain data, wearable physiological measurement systems and modelling. We see the future of measuring behavior research being especially linked to developing more robust modelling and data analysis techniques to help improve movement tracking and classification, since this a common theme through many of these papers. We can see that work of this nature could encourage sport to become safer, and also less subjective, in terms of judging and training. A better understanding of human behavior will also promote more robust studies by acknowledging participant and session variability.

We hope that this collection will stimulate further research developing behavioral measurement systems in sport and exercise, and the continued development of associated devices and techniques. We see many opportunities in this area, and welcome more submissions on the topic of measuring behavior to Sports Engineering in the future. We would especially like to see field studies on the effect of equipment interventions that have many participants and highly accurate measurement techniques (see Topical Collections on Football Research, Surf Engineering and Winter Sports ${ }^{1}$.

\section{Reference}

1. Grant RA, Allen T, Spink A, Sullivan M (2018) Measuring Behavior 2018 Conference Proceedings. https://www.measuringbehavi or.org/mb2018/proceedings.html

Publisher's Note Springer Nature remains neutral with regard to jurisdictional claims in published maps and institutional affiliations.

\footnotetext{
$\overline{1}$ https://link.springer.com/journal/volumesAndIssues/12283?tabNa $\mathrm{me}=$ topicalCollections.
} 